\title{
Estructura de Gestión basada en el proceso S\&OP: Estudio de caso en una industria cosmética Brasileña
}

\section{(Corporate governance structure which is based in the S\&OP process: Case study in la Brazilian cosmetic company)}

\author{
Ximena Rojas ${ }^{1}$, Luciana Hazin ${ }^{2}$
}

\begin{abstract}
Resumen:
El articulo trata de la generación de una estructura de gestión empresarial basada en el Proceso S\&OP en una industria cosmética Brasileña. El creciente énfasis del desarrollo empresarial, guiado por la búsqueda de la atención a las necesidades y exigencias de los clientes está haciendo que organizaciones de todos los sectores trabajen en la creación de un diferencial competitivo. La estrategia de gestión empresarial colaborativa S\&OP atrae cada vez más la mirada de los interesados que buscan potencializar sus prácticas para alcanzar este diferencial. Con la metodología estudio de caso se busca conocer, identificar y analizar las características de la empresa, usando múltiples herramientas de análisis (SWOT, Análisis de procesos, Cadena de valor) a fin de proponer una herramienta de gestión con bases sólidas que se ajuste a las necesidades y objetivos propios de la organización. Los resultados demostraron que los principios de colaboración y comunicación internas son los pilares fundamentales frente a cualquier intento por el desarrollo empresarial; esto sustenta la propuesta de una estructura de gestión basada en los cinco pasos del Proceso S\&OP; como complemento al estudio se identifica al modelo CPFR como plataforma para un trabajo integrado con las demás empresas de la Cadena de Suministros.
\end{abstract}

Palabras clave: Proceso S\&OP; Gestión empresarial; Diferencial competitivo; Colaboración; Cadena de Suministros.

\begin{abstract}
:
This article is about the generation of a corporate governance structure which is based in the process S\&OP for a Brazilian cosmetic industry. The increasing emphasis on business development guided by attention to the requirements and demands of customers, cause nowadays, that the organizations of the commercial sectors work in the creation of a competitive differential. The collaborative business management strategy S\&OP is looking for the attention of people who are interested in the improvement of their skills; in order to achieve this differential. With the case study methodology, the objectives are to get knowledge, identify, and analyze the characteristics of the company using analysis tools like SWOT, process analysis, value chain. All with the purpose to get a robust management tool that fits with the requirements and demands of the organization. The analyzed results showed that the principles of collaboration and internal communication were the pillars necessary against any attempt of business development. This supports the proposal of a management structure based on the five step S\&OP process. As a complement to this study a methodology CPFR was identified as the platform for an integrated work with the rest of the companies of the supply chain.
\end{abstract}

Keywords: Process S\&OP; business management; competitive differential; collaboration; supply chain.

\footnotetext{
${ }^{1}$ Universidad Tecnológica Equinoccial, Ingeniería Industrial, Quito - Ecuador (rlxb92844@ute.edu.ec)

${ }^{2}$ Universidade federal de Pernambuco, Engenharia de Produção, Recife - Brasil (Ihazin@ufpe.br)
} 


\section{Introducción}

Varios factores han contribuido para el crecimiento del Sector Cosmético en el Brasil y en el mundo entero, entre los cuales se destaca la participación creciente de la mujer brasileña en el mercado de trabajo, la utilización de tecnología de punta y el consecuente aumento de la productividad, el lanzamiento constante de nuevos productos en la búsqueda de una mejor y más precisa atención a las necesidades del mercado. Esta evolución acelerada del mercado está obligando a la Industria Cosmética a potencializar sus procesos, gestionar efectivamente el trabajo diario y aplicar estrategias competitivas más eficaces que les permitan evolucionar ordenada y firmemente dentro del sector que se muestra cada vez más competitivo. Con desarrollo, innovación y una buena dosis de acción socio ambiental, el sector cosmético poco a poco asume una producción que demuestra el cuidado en todas las etapas de la concepción del producto (Planificación, marketing, diseño, desarrollo, producción y control de calidad) lo que notoriamente está generando resultados mundiales.

De modo general, las organizaciones envueltas en el sector cosmético conviven con un ambiente dinámico y exigente lo que demanda de ellas una constante innovación, diversidad productiva y fluidas estrategias comerciales y de marketing, con el objetivo de llevarlas a los más altos niveles de liderazgo empresarial. La herramienta empresarial colaborativa se está tornando en una aliada estratégica para el desarrollo empresarial buscado, este nuevo entendimiento está haciendo que muchas organizaciones intenten ganar un diferencial competitivo a través de la integración de sus procesos y negocios. La estrategia empresarial S\&OP llega con el objetivo de alcanzar la excelencia operacional y la innovación de los modelos de negocio en las organizaciones.

El objetivo de este trabajo es proponer una estructura de gestión basada en el Proceso S\&OP y analizar su aplicación en una empresa cosmética localizada en la ciudad de Recife, estado de Pernambuco, para ello se presenta a la empresa el respaldo suficiente y necesario que le permita incursionar en la fusión de su acelerado crecimiento empresarial y sus evidentes necesidades de comunicación y colaboración con los beneficios que este proceso colaborativo brinda.

Para diseñar la propuesta de trabajo, se aprovecha de la orientación entregada por la metodología estudio de caso. Según (Yin, 2010), la importancia del protocolo de estudio de caso está en mantener el enfoque sobre el tópico del estudio. Conforme (Yin, 2010), la colecta de evidencia viene de varias fuentes como: documentación, registros en archivo, entrevistas, observación directa e indirecta. En este caso particular se hace uso de las entrevistas, la observación directa, y del análisis de la documentación interna; y de acuerdo a lo expuesto por (Cauchick, 2007) se construye un referencial teórico conceptual a fin de mapear los documentos bibliográficos sobre el asunto de la investigación. 


\section{Metodología}

\subsection{Proceso de Planificación de Ventas y Operaciones "S\&OP" (Sales and Operation Planning)}

De acuerdo con (Bremer, Azevedo, \& Matheus, 2008) un modelo de gestión basado en el Proceso de S\&OP que reconozca y trate de forma distinta diferentes cadenas de atención de la empresa consiste en una de las principales tendencias actuales. Además, la aplicación de la S\&OP global e integración del proceso con clientes y proveedores son tendencias que ratifican la creciente búsqueda por una visión más amplia de las Cadenas de Suministro.

Acogiendo lo destacado por (Navarro \& Lima, 2006), dentro del mercado de cambios frecuentes, es cada vez más urgente la necesidad de las empresas de contar con el apoyo de técnicas de planificación y sistemas integrados de gestión que les permitan alcanzar las metas establecidas en la estrategia corporativa, de frente a esta realidad el S\&OP está ganando reconocimiento creciente.

De acuerdo con (APICS, 2000) se puede afirmar que los beneficios obtenidos con una implementación eficaz del S\&OP son:

> Para las empresas de fabricación para inventarios: Alto nivel de atención al cliente y bajos inventario de productos acabados, simultáneamente;

> Para las empresas de fabricación bajo pedido: Alto nivel de atención al cliente y frecuente bajo lead time de atención al, al mismo tiempo;

> Una producción más nivelada sin picos o valles, reducción de horas extras e

$>$ y aumento de productividad;

> Reducción de conflictos entre las áreas de ventas, marketing, finanzas, producción, logística, planificación de materiales, y desarrollo de productos.

\subsection{Modelo Uno - S\&OP}

Para (Wallace, 2001), la esencia del S\&OP es la toma de decisiones para cada familia de producto, que determinan el cambio, actualización o creación de planes de ventas, operaciones, inventarios y de pedidos pendientes o atrasados. Después de la aprobación de estos planes por el equipo del S\&OP ejecutivo, estos pasan a ser documentados y distribuidos para toda la organización. El conjunto de estos planes es el plan de acción global para las áreas de ventas (Marketing y Comercial), operaciones, finanzas y desarrollo de productos. El paso siguiente es la generación de planes agregados del S\&OP, en los niveles de detalle necesarios o requeridos por cada área. 
En la Figura 1 se presentan las etapas e informaciones que conforman el Proceso S\&OP.

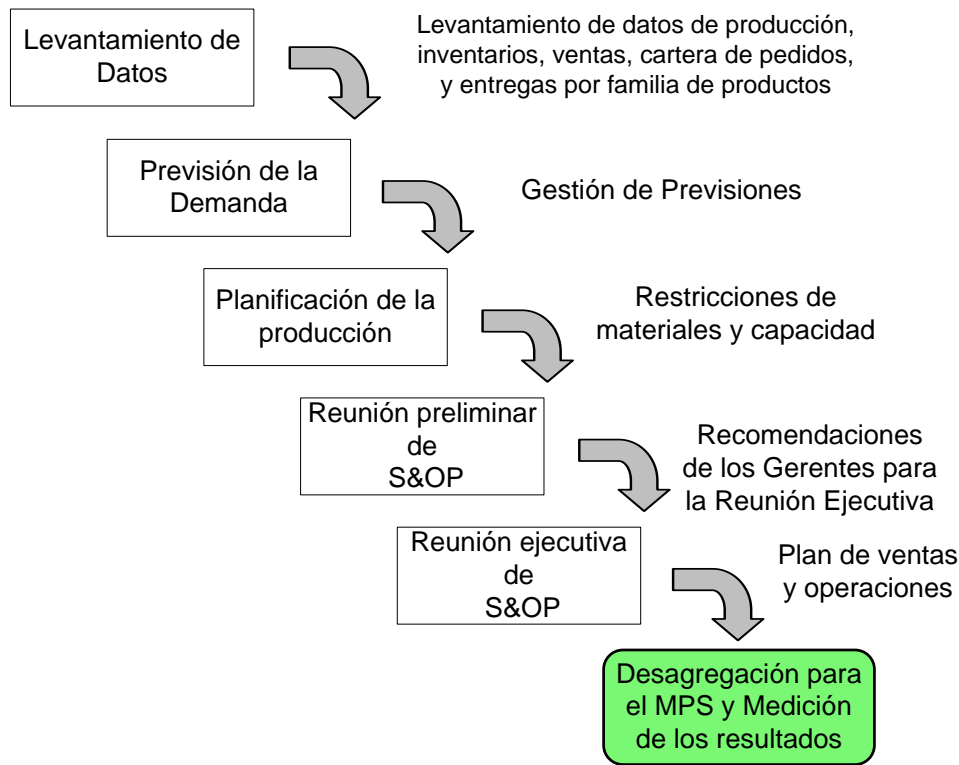

Figura 1. Etapas del Proceso S\&OP

Fuente: Adaptado de Wallace (2001)

El levantamiento de datos parte de la revisión de archivos actualizados del mes reciente. El área de Ventas usa esta información en la preparación de la nueva previsión.

Para la planificación de la demanda, el personal de ventas recibe, analiza y discute la información proveniente del Paso 1, ajusta la nueva previsión para productos existentes así también como para los nuevos productos. El resultado de la planificación de la demanda es la previsión que va a ser sometida a la autorización de la gerencia.

En la Planificación de los suministros (Capacidad), se hace la modificación de los planes de operaciones, a causa de los cambios en la previsión de ventas. El producto de esta fase es el Plan de Suministros en el cual se reflejan los cambios, restricciones, acciones de contingencia y priorización, con el objetivo de dar atención al plan de la demanda.

La reunión preliminar S\&OP, tiene los siguientes objetivos:

- Tomar decisiones con relación a la fluctuación de la oferta y la demanda;

- Definir soluciones a los problemas y diferencias surgidas en las etapas anteriores con el fin de determinar un conjunto único de recomendaciones a ser llevadas a la reunión del S\&OP Ejecutiva;

- Identificar las áreas donde el consenso no puede ser alcanzado, y determinar la forma de presentación de aquellos puntos en la reunión S\&OP Ejecutiva;

- Definir la pauta de la reunión del S\&OP Ejecutiva.

- Los participantes de esta reunión son profesionales de las áreas de: Planificación de la Demanda, Investigación y Desarrollo, Suministros, Finanzas, y el director del proceso S\&OP. 
En la reunión del S\&OP Ejecutiva, se pretende alcanzar lo siguiente:

- Tomar decisiones para cada familia de productos;

- Aprobar los cambios de los planes de operación o adquisición;

- Relacionar la versión financiera de las informaciones del S\&OP con el Plan de Negocio y realizar los ajustes necesarios;

- Dar solución a los dilemas presentados por el equipo que sesionó de forma preliminar;

- Tomar decisiones en cuanto a la atención al cliente, desarrollo de nuevos productos y proyectos especiales, etc.

Los resultados de esta fase son los registros de la reunión, con el detalle de las decisiones tomadas, definición de acuerdos, especificación de las modificaciones del plan de negocio.

\subsection{Modelo Dos - S\&OP/IBP}

S\&OP ha evolucionado a Integrated Business Planning - IBP, donde todos los planes de la empresa: de producto, de demanda, de oferta, iniciativas estratégicas y financieras son sincronizadas y alineadas al nivel agregado de cada mes (Aberdeen Group, 2008). S\&OP/IBP es mucho más que un Proceso táctico o de integración de la CS, permite gestionar un negocio de forma integrada y holística (Schorr, 2007). S\&OP/IBP minimiza o elimina las lagunas o gaps. El énfasis es administrar el negocio de forma holística. La Figura 2 ilustra el ciclo mensual del Proceso S\&OP/IBP estructurado en cinco pasos.

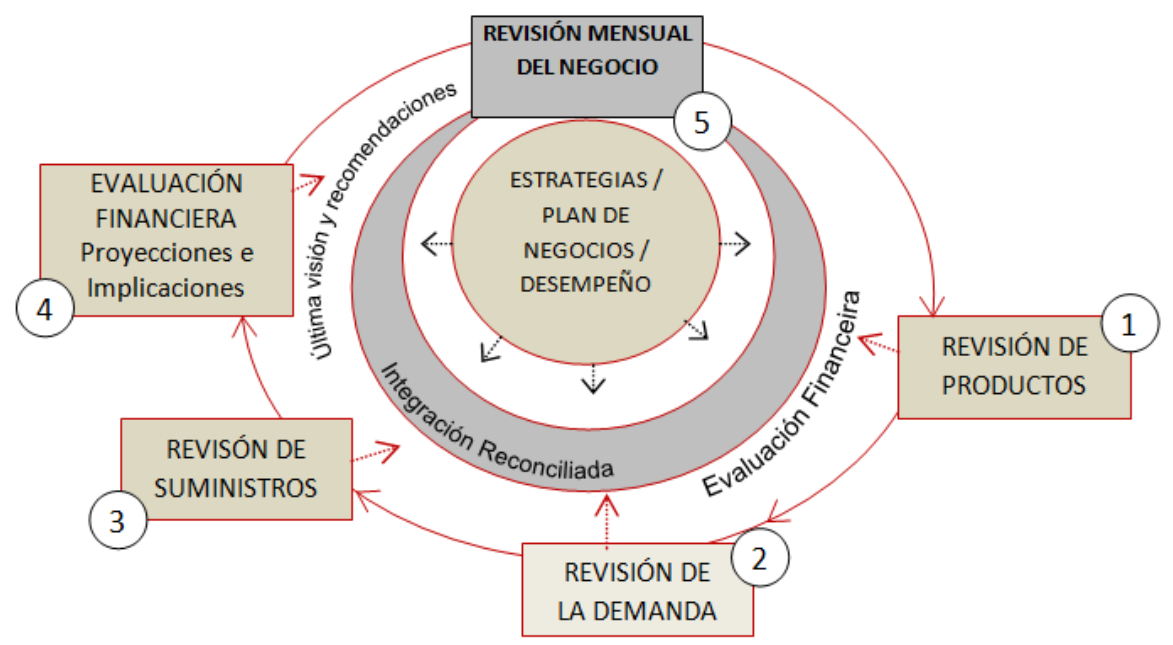

Figura 2. Planificación Integrada de Negocios (S\&OP/IBP): Cinco pasos del Proceso Fuente: (Palmatier \& Crum, 2003)

La gestión de productos y nuevas actividades revisa el portafolio de los productos y de las nuevas actividades que influencien la demanda y el suministro. El resultado es un plan con la evaluación de los recursos para la implantación de las nuevas actividades. 
La gestión de la demanda revisa los planes de demanda de todos los productos de la empresa y de los factores que los sostienen. Los planes de demanda son resumidos de forma agregada, por familia y subfamilia.

La habilidad de suministro de la CS para sostener el plan de nuevas actividades y el plan de demanda, es validado por la gestión del suministro. Se revisan las alternativas de materiales, capacidad de producción, restricciones, flexibilidad, planes de mejora y patrones estacionales.

Para la revisión integral y evaluación financiera se usan los planes desarrollados en los pasos anteriores, generando un plan integrado de negocio. El resultado de la S\&OP será encaminado al equipo de gestión para la toma de decisiones finales.

Finalmente, en la revisión mensual se generará un conjunto único de planes integrados, una evaluación total de la planificación del negocio para los próximos 18-24 meses, que deberá ser comunicada inmediatamente a toda la organización.

Ejecutado correctamente el Proceso de S\&OP el equipo de gestión puede actuar de forma estructurada, ágil y colegiada, con base en la comunicación.

\subsection{Descripción de la empresa}

La empresa en estudio forma parte de un grupo de negocios formado por tres empresas que realizan sus actividades en el campo cosmético. Su finalidad es el desarrollo, producción y entrega de dermocosméticos, también conocidos como cosméticos de tratamiento; las actividades de gestión y productivas son desarrolladas en Brasil, en la región Nordeste en el estado de Pernambuco, con base en la ciudad de Recife, atendiendo a clientes de todo el país.

En la actualidad, la empresa se encuentra en una etapa de madurez, en gran parte forzada por los acentuados cambios del sector a consecuencia de las exigencias y expectativas de sus clientes; a esto se suma el reflejo del crecimiento empresarial como respuesta al eco económico mundial. Con esto, es visible la necesidad de incorporar la mejor propuesta de gestión empresarial que fortalezca sus operaciones internas y que sirva de plataforma expandir positivamente la eficiencia y efectividad de sus actividades hacia el resto de empresas que componen la Cadena de Suministros en la cual se encuentra incluida.

\subsection{La problemática}

La empresa reconoce la importancia de contar con bases sólidas para el gerenciamiento empresarial que serán de utilidad para alcanzar los propósitos de reconocimiento y posicionamiento nacional e internacional. La generación y usufructo de una ventaja competitiva sustentable y permanente será la distinción del proceder diario de la empresa. 
De la evaluación de procesos de los niveles estratégico e táctico, se encontraron los siguientes indicativos:

- Proyectos con plazos de ejecución expandidos (fuera de la fecha de compromiso);

- Escasa evidencia de la evaluación de los indicadores de desempeño que reflejen la medición a los proyectos ya ejecutados.

- La compañía presenta una estructura tradicional que no acompaña el crecimiento empresarial expandido; las relaciones de comunicación y colaboración se ven impactadas.

- Utilización de varios sistemas de información, que se muestran competentes en las áreas que los requieren, sin embargo presentan dificultades en el momento de su integración y al compartir datos; este hecho obliga a que los procesos realicen re trabajos o actividades manuales.

- El Proceso administrativo-financiero; presenta dificultades para procesar los resultados de los indicadores de desempeño, la unión de la planificación estratégica con las planificaciones táctica y operativa no se establece apropiadamente.

En este contexto la empresa precisa trabajar sobre un proceso de gestión que permita el desarrollo hacia un direccionamiento estratégico, sustentado en procesos fuertes, integrados y colaborativos con el apoyo de un sistema informático integral.

\section{Resultados}

La aplicación del Proceso S\&OP asegura sincronía de los planes tácticos de los Procesos, la Figura 3 presenta el posicionamiento de los Procesos de la empresa dentro de la estructura global del negocio, así también como el vínculo con el Proceso S\&OP.

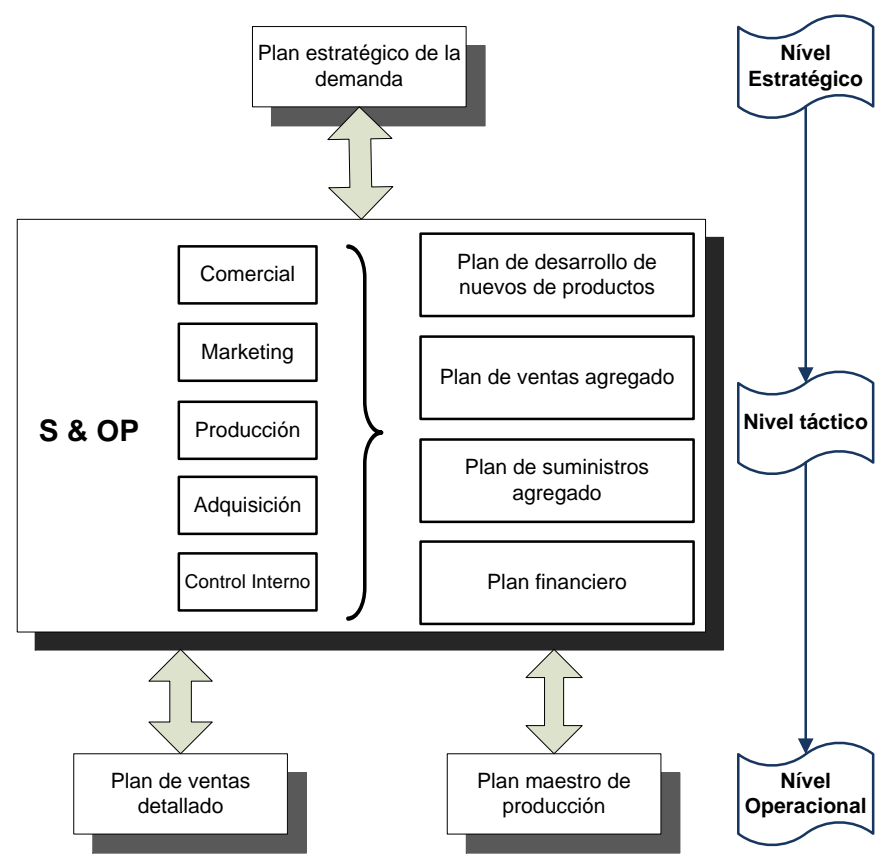


Figura 3. Vínculo de los Procesos de la empresa con el Proceso S\&OP con la estructura global del negocio

Fuente: (Corrêa, Gianesi, \& Caon, 2001)

La descripción de los cinco pasos del Proceso S\&OP toma como referencia el panorama actual de la empresa y sobre este se aportan sugerencias de mejora para el fortalecimiento de cada paso.

La visión inmediata de la estructura de gestión con el Proceso S\&OP es la implementación y permanencia a través de la mejora continua, por ello; es conveniente acoplar a la propuesta, la designación de responsables que conduzcan cada fase, lo que permitirá organizar y encaminar el proyecto.

La generación de la estructura de gestión comienza por la identificación del Líder del proyecto, según (Wallace, 2001) la mejor elección es el Presidente o Director de la empresa, el entusiasmo e iniciativa para llevar a la empresa a fusionar las actividades cotidianas con la esta estrategia de gestión empresarial parte de la cabeza líder de la empresa.

(Wallace, 2001) Considera importante la existencia de un "director del Proceso, quien gestione el proyecto de implementación" del Proceso S\&OP.

\subsection{Levantamiento de los Datos}

Se trabaja en las principales fuentes (actividades del proceso productivo). Al final del mes, se efectúa el análisis de los inventarios de materia prima, insumos, producto en proceso y producto terminado; el reporte generado presenta la necesidad de compra de materia prima e insumos, identificación y cantidad de producto en inventario, comunicación de la racionalización de productos (poca demanda de los mismos), pendencias de productos a ser entregados a los clientes.

De la primera evaluación al levantamiento de la información, se tiene que la empresa realiza de forma satisfactoria esta etapa, sin embargo, el tiempo para la consolidación de los datos en planillas electrónicas y su posterior comunicación vía e-mail, encontraría un mejor aprovechamiento con el uso de un sistema de información integrado.

En esta fase del proceso S\&OP, el desarrollo de nuevos productos debe estandarizarse en un POP y su respectivo informe, el objetivo es la conducción de la información de una forma consolidada, refiriéndose a las nuevas $u$ obsoletas actividades, recursos y cambios que la implementación de productos nuevos traen a los procesos.

\subsection{Planificación de la Demanda}

La empresa desarrolla un conjunto de acciones para planificar la demanda, que al comienzo de la tarea se muestra eficaz al juntar el trabajo colaborativo de los equipos de Ventas y Operaciones. 
El equipo operativo desarrolla una primera previsión de demanda fundamentada en los dados históricos de salida de los productos ${ }^{3}$, que es complementada con la información obtenida por el equipo comercial, proveniente de la captación de las tendencias y preferencias del mercado, de allí que resulta imperativa la existencia de un Proceso formal para el desarrollo de nuevos productos.

En cuanto a las acciones colaborativas efectuadas en esta etapa, se hace necesario trabajar en la mejora continua. A continuación se presentan algunas sugerencias para perfeccionar esta etapa:

- Trabajar con un proceso padrón para el desarrollo de nuevos productos que permitirá originar ganancias importantes para la organización: los envueltos en el proceso conocen sus funciones y atribuciones, los tiempos de respuesta entre departamentos se verían reducidos debido al compromiso en la agilidad de respuesta, en primer lugar con el cliente, y segundo, con sus compañeros de trabajo, la meta financiera sería alcanzada en menor tiempo y finalmente el acompañamiento productivo ofrecería mejores niveles de cumplimento, de acuerdo con (Ireland \& Crum, 2006) los requerimientos de clientes y colegas comerciales se constituyen en el primer enlace establecido entre los procesos S\&OP y CPRF.

- Integrar el sistema de información, una adecuada divulgación de procesos trae consigo innumerables beneficios, a este se suma otro que se refiere al producto resultante de esta etapa "La nueva previsión autorizada por el Directorio". El rápido acceso a la información permitiría alcanzar este resultado en menor tiempo y con la garantía de trabajar con datos precisos.

- Incluir los cuatro principios básicos para la generación del plan de demanda presentados en la propuesta, así como para su gestión; esto pretende direccionar el camino para alcanzar un plan de demanda con acciones priorizadas.

- Es conveniente estructurar un equipo permanente que trabaje focalizado en la planificación de la demanda, este equipo está formado por personas de las áreas de ventas, atención al cliente, contabilidad, desarrollo de nuevos productos y el "director del Proceso"; la cantidad de miembros dependerá de los requerimientos y disponibilidad de la empresa.

\subsection{Planificación de los Suministros}

En esta etapa se toman decisiones críticas con respecto a la posición del inventario y a la capacidad de producción disponible en la cadena. Los autores (Vieira \& Neumann, 2008) afirman que la etapa de gestión de los suministros comienza con el plan de la demanda. El resultado de esta etapa es el plan de suministros en el cual se reflejen los cambios, restricciones, acciones de

\footnotetext{
${ }^{3}$ La característica operativa de la empresa es la fabricación de productos para inventario, lo que significa
} que la empresa conoce sus clientes a través de la Planificación de la demanda y la Previsión de las ventas. 
contingencia y priorización; este plan aborda las tareas a ser desarrolladas por las áreas operativas: producción (logística interna, fabricación y logística externa) y adquisición.

A seguir en la Tabla 1 se presenta el análisis SWOT (Fortalezas, Debilidades, Oportunidades de mejora y Amenazas) realizado al proceso productivo, a fin de sintetizar la información levantada en el estudio.

Tabla 1- Análisis SWOT del Proceso Productivo de la empresa en estudio

\begin{tabular}{|c|c|}
\hline ANÁLISIS SWOT & PROCESO PRODUCTIVO \\
\hline Fuerzas & 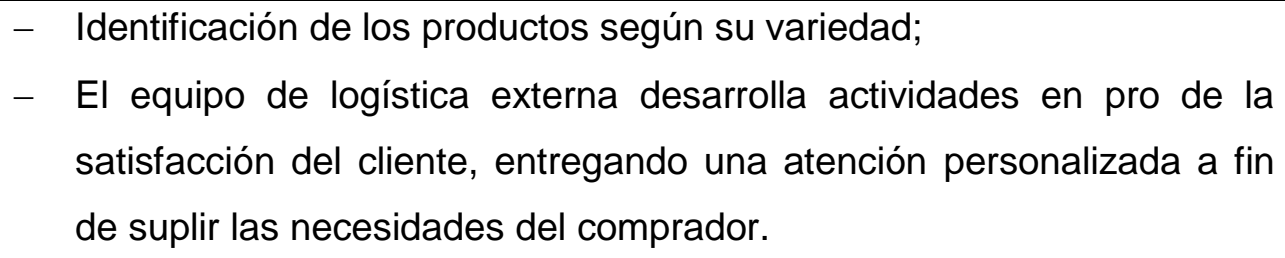 \\
\hline Debilidades & $\begin{array}{l}\text { - La informalidad de los cambios en el portafolio de productos, sumado } \\
\text { al deficiente sistema de información y comunicación, trae serios } \\
\text { inconvenientes para los demás procesos; } \\
\text { - La responsabilidad de la actualización de datos por cuenta del } \\
\text { subproceso de logística interna demanda vigilancia constante de los } \\
\text { procesos líderes (adquisición y producción), se trata de una actividad } \\
\text { en gran parte manual. }\end{array}$ \\
\hline $\begin{array}{l}\text { Oportunidades } \\
\text { de mejora }\end{array}$ & $\begin{array}{l}\text { - La etapa de planificación de suministros se vería mejorada con la } \\
\text { definición de un equipo que, mes a mes, trabaje focalizado en esta } \\
\text { tarea; } \\
\text { - } \text { Realizar una agregación jerárquica de productos; } \\
\text { _ Incluir dentro del POP la actividad que procese adecuadamente los } \\
\text { cambios en el portafolio de productos (alteraciones, racionalización, } \\
\text { productos descontinuado, etc); } \\
\text { - Definir y divulgar un plan de operaciones mensual; } \\
\text { - Desarrollar modelos de gestión para atender la planificación de } \\
\text { producción (flujo de producción, requerimientos y restricciones). }\end{array}$ \\
\hline Amenazas & $\begin{array}{l}\text { - Disminución de la productividad debido a la necesidad de un Sistema } \\
\text { de Información Integrado. }\end{array}$ \\
\hline
\end{tabular}

Es preciso trabajar en propuestas para dar atención la estos cuatro aspectos que están siendo de impacto en el proceso productivo. Se considera conveniente que el equipo gestor de la planificación de los suministros, esté conformado por los gerentes de producción, contabilidad, desarrollo de nuevos productos y el "director del proceso S\&OP". 
En esta fase de la propuesta se encuentra conveniente agrupar los productos y recursos en tres categorías: ítems, tipos de productos y familias. Dentro de los ítems se encuentran los productos finales, dentro de los tipos de productos se alojan los grupos de ítems que poseen estructura de costos, procesos productivos y agrupamiento semejantes; y por último, dentro de las familias están los grupos de ítems pertenecientes a un mismo tipo de producto y que comparten tiempos de preparación semejantes.

Las ventajas más destacables de esta práctica comienzan por la reducción de las complejidades en todas las etapas del proceso S\&OP (se consigue realizar previsiones de demanda más precisas para luego enseguida ajustarse el plan de operaciones de tal manera de dar atención a la demanda en cuestiones de inventario, volumen y mix de pedidos en los niveles deseados), reducción de la incerteza en el momento de la planificación agregada (permite que los planes de operaciones y de capacidad trabajen con mayor precisión, identificando recursos y capacidades necesarios) y; finalmente la mayoría de las empresas realiza la planificación de su negocio con base en la jerarquía de los productos.

Íntimamente ligada a la jerarquía e identificación de los productos, se encuentra el descontinuar productos; la realización de esta actividad es efectuada cuando es necesaria, sin embargo el complemento documental todavía precisa ser instaurado. La inclusión de esta actividad en el POP va a traer consigo la formalización de la operación, y la mejor comunicación de los procesos, cuando los motivos para tal acción provengan de otras fuentes que no sea el área de producción.

También, se precisa de un plan de operaciones con el nivel apropiado de flexibilidad que atienda a los cambios hechos por el proceso de ventas. La divulgación formal de este plan permite a los procesos operativos actuar con eficiencia y evitar la presencia de imprevistos.

De esta fase de planificación de los suministros surgen informes que reúnen datos de capacidad operativa, restricciones y necesidades en cuanto al cumplimiento de pedidos, para ello se propone la creación de un informe consolidado donde se evidencie el desarrollo de modelos de optimización enfocados a actuar frente a las eventualidades que salen del padrón de producción. Los problemas encontrados en la planificación, así como las respectivas propuestas para su tratamiento son parte de las informaciones que el equipo de operaciones van a llevar a la reunión preliminar S\&OP.

Compartir responsabilidades e informaciones sobre las necesidades del cliente y de los demás procesos ha favorecido a la expansión del papel de la adquisición que hoy en día atiende niveles de coordinación y desarrollo de proveedores, investigación del mercado de proveedores, análisis de costos, formulación de la estrategia de tercerización, benchmarking, poder en la decisión entre hacer o comprar; y el análisis de la capacidad del proveedor.

La contribución general que esta etapa hace al resto del proceso es la identificación de los problemas de suministros que no pudieran ser resueltos por el equipo y que serán agregados a la 
lista y llevada por operaciones, para la reunión preliminar S\&OP (paso siguiente). Ejemplos de estos problemas pudieran ser: aprobación de mayores cantidades de compra de algún insumo, o material específico requerido, evaluación de nuevos acuerdos de negociación frente a la necesidad de compra de nuevos proveedores, contratación del recurso humano, etc.

La Figura 4, presenta la relación de la planificación de la demanda y de los suministros, así también como las entradas y salidas de cada proceso y la contribución para la planificación empresarial.

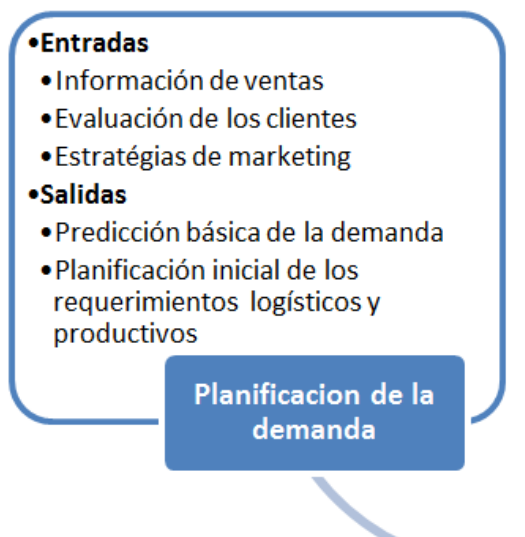

\section{-Entradas}

- Estratégias de marketing

- Predicción básica de la demanda

- Planificación inicial de los

requerimientos logísticos y

Planificacion de la demanda
Planificación de los

suministros

- Predicción de la demanda

- Restricciones empreariales y de la CS

-Salidas

-Planificación de la producción

- Planificación de los suministros

- Planificación de la logística

Figura 4. Planificación de la Demanda y Planificación de los Suministros, alineados para la Planificación Empresarial

Fuente: (Bowersox, Closs, \& Cooper, 2007)

\subsection{Reunión de preliminar S\&OP}

Antes de las últimas dos fases del Proceso S\&OP, gran parte de las actividades definidas para estas etapas se encontraban encaminadas y en desarrollo. En este caso, se consideran pertinentes los siguientes aspectos que pudieran ser de utilidad para el mejoramiento de las actividades.

La efectividad, productividad y rendimiento de los resultados de esta etapa dependerán de la calidad de la información entregada por los pasos anteriores.

Para la organización, se encuentra conveniente que los principales participantes de esta reunión sea en principio los gerentes de los procesos de: marketing, comercial, producción, adquisición y contraloría, por el conocimiento que cada gerente tiene de su proceso, lo que va a traer garantía de los resultados en las decisiones tomadas en forma colegiada.

Los resultados esperados de la reunión preliminar S\&OP serían: 
- La definición de los indicadores para medición del desempeño del proceso S\&OP (resultado de la primera reunión), para el acompañamiento de los planes de Ventas, operaciones, inventario, cumplimiento de pedidos y atención al cliente.

- Con los resultados obtenidos de la medición del desempeño, se define un cronograma mensual, trimestral y semestral para la verificación de la coordinación entre los planes estratégico, táctico y operacional de la empresa.

- En forma colegiada, decidir la mudanza, restricción y criterios para descontinuar productos, de tal forma que la toda empresa tenga conocimiento y acceso a la misma fuente de información.

- El acompañamiento y validación del proceso de desarrollo de nuevos productos, para garantizar el éxito del nuevo lanzamiento.

- La mejora en la comunicación interna y externa, una vez que todos los procesos se enlazan en una sola fuente de información por la que fluyen decisiones y estrategias empresariales.

- La evaluación de las propuestas sugeridas por las áreas de ventas y operaciones, como posibles pautas de solución a los problemas también deberán ser presentada en esta reunión.

- Finalmente, también será generado un esbozo con los puntos a ser tratados en la reunión ejecutiva S\&OP.

\subsection{Reunión Ejecutiva del S\&OP}

El equipo de S\&OP Ejecutivo deberá estar compuesto por los gerentes de los Procesos claves, el director del proceso y la persona responsable por el Directorio.

Esta reunión tendrá características de mayor liderazgo que la reunión preliminar S\&OP, los resultados a ser obtenidos de este paso se distinguen, particularmente, por su distinción decisoria y aprobadora ante los pontos presentados de la fase anterior. Se entiende que los aspectos aquí tratados no alcanzaron una solución apropiada durante el caminar de las cuatro fases precedentes, o son de tal transcendencia que solo pudieran ser atendidos en esta fase del proceso.

\section{Discusión}

El mundo contemporáneo de ágil crecimiento empresarial está motivando a las empresas de todos los sectores a envolverse en actividades y estrategias de gestión que maximicen su potencial individual y promueva el fortalecimiento de las relaciones colaborativas. La propuesta de estructura de gestión basada en el Proceso S\&OP actúa sobre las áreas de Ventas y Operaciones de la empresa con el objetivo de fortalecer a la compañía con los lineamientos de un proceso de 
gestión empresarial integrado, fundamentado en los principios de la colaboración y comunicación internas.

La propuesta toma como base los modelos de gestión propuestos por (Wallace, 2001) y (Palmatier \& Crum, 2003), mismos que son mundialmente conocidos por los resultados alcanzados. La característica que los datos mostraron al Estudio de caso como la estrategia de investigación que se ajustaba al alcance de los objetivos pretendidos; y a partir de esto fue posible realizar un análisis completo de la organización y sus requerimientos empresariales. En la propuesta presentada, fueron identificadas las fases del proceso y las respectivas propuestas de mejora que pudieran llegar a viabilizar de manera eficiente el proceso.

La principal limitante identificada en el estudio es la aplicación de esta estructura en otras empresas, a pesar de ser del mismo ramo empresarial, se precisaría de un estudio igual o hasta mismo más profundo del que se ha desarrollado aquí. La validad externa que hace referencia a la generalización de los resultados alcanzados en el estudio es la gran limitante del trabajo, la aplicación de los mismos resultados en otras organizaciones empresariales deberán pasar por un proceso de prueba. Finalmente, la investigación de la realidad de la Cadena de Suministros que envuelve a la empresa (empresa central) pudiera ser el paso siguiente para estudios todavía más complejos y robustos como el Proceso CPFR.

\section{Conclusiones y Recomendaciones}

- Con el análisis de la situación de la empresa cosmética en ese momento fue posible reconocer sus fortalezas y debilidades cada Proceso, y con esto fue definida la línea base para el comienzo del estudio y el desarrollo de la propuesta.

- El Proceso S\&OP a través de cada uno de sus pasos ofrece una metodología sistemática para el trabajo colaborativo de los procesos internos empresariales; cuando la visión de crecimiento empresarial es alcanzar una gestión de la cadena de suministros efectiva.

- El éxito de la metodología S\&OP, está cuando se reconoce la importancia de la colaboración y trabajo conjunto; donde los resultados dependen del flujo eficiente de la información para una planificación empresarial sincronizada.

- El desarrollo de las actividades empresariales basado en el modelo S\&OP busca generar una fuerte cultura organizacional que entregue un soporte para proyectos de gestión todavía más vigorosos, como es CPFR, donde el trabajo colaborativo excede los límites internos. 


\section{Bibliografía}

Aberdeen Group. (2008). Enterprise Sales and Operations Planning, 2008. Retrieved Junio 10, 2010, from http://www.aberdeen.com/

APICS. (2000). Management, The Educational Society for Resource Master Planning of Resources (version 1.0 June 2000). Virginia.

Bowersox, D., Closs, D., \& Cooper, M. (2007). Administración y logística en la cadena de suministros. México.

Bremer, C., Azevedo, R., \& Matheus, L. (2008). O retrato do processo de Sales \& Operations Planning (S\&OP) no Brasil - Parte 1,2,3. Revista Mundo Logística, 10-16.

Cauchick, M. (2007). Estudo de caso na engenharia de produção: estruturação e recomendações para sua condução. Produção, pp. 216-229.

Corrêa, H., Gianesi, I., \& Caon, M. (2001). Planejamento, Programação e Controle da Produção; MRP II / ERP - Conceitos, Uso e Implantação. São Paulo: Atlas.

Ireland, R., \& Crum, C. (2006). Linking CPFR to S\&OP. The Oliver Wight - White Paper Series.

Navarro, J., \& Lima, R. (2006). Planejamento de vendas e operações (S\&OP): um estudo de caso em uma empresa da indústria de telecomunicações. XXVI ENCONTRO NACIONAL DE ENGENHARIA DE PRODUÇÃO - ENEGEP. Fortaleza.

Palmatier, G., \& Crum, C. (2003). Enterprise Sales and Operations Planning: Synchronizing Demand, Supply and Resources for Peak Performance. J. Ross Publishing Integrated Business Management Series.

Schorr, J. (2007). Integrated business management. Business Excellence.

Vieira, D., \& Neumann, D. (2008). Melhores Práticas - Preparando-se para a colaboração "Gestão da demanda e S\&OP". Revista Mundo Logística, 1(5): 45.

Wallace, T. (2001). Planejamento de Vendas e Operações: guia prático "S\&OP" (Sales \& Operations Planning). São Paulo: Instituto IMAM.

Yin, R. K. (2010). Estudo de Caso - Planejamento e Métodos. Porto Alegre: Bookman. 